\title{
Variability of Serum Concentration of Calcium, Phosphate and Parathyroid Hormone Depending on Time of Blood Draw for Patients on Nocturnal Home Hemodialysis*
}

\author{
Nasim Shahbazi ${ }^{1}$, Pierre A. Brown ${ }^{2}$, Ayub Akbari ${ }^{2,3}$, Deborah L. Zimmerman ${ }^{2,3}$ \\ ${ }^{1}$ Department of Medicine, Division of Nephrology, Thunder Bay Regional Health Sciences Centre, Thunder Bay, Canada \\ ${ }^{2}$ Department of Medicine, Division of Nephrology, Ottawa Hospital, Ottawa, Canada \\ ${ }^{3}$ Kidney Research Centre, Ottawa Hospital Research Institute, Ottawa, Canada \\ Email: dzimmerman@ottawahospital.on.ca
}

Received August 17, 2012; revised September 23, 2012; accepted October 7, 2012

\begin{abstract}
Background: Guidelines for patients treated with conventional hemodialysis patients have been written for target serum levels for calcium (Ca), phosphate $\left(\mathrm{PO}_{4}\right)$ and intact parathyroid hormone (iPTH). No guidelines exist for nocturnal home hemodialysis (NHHD) patients for target values or timing of the blood sample draw. We undertook a prospective cohort study to examine the variability in pre, post and clinic (post-post) serum values for $\mathrm{Ca}, \mathrm{PO}_{4}$, and iPTH in NHHD patients to determine if timing of blood draw could affect clinical decisions. Methods: Twenty prevalent NHHD patients collected blood pre and post their usual NHHD session with an additional blood sample drawn in clinic (postpost). Median and interquartile range of pre, post and clinic (post-post) values of $\mathrm{iPTH}, \mathrm{PO}_{4}$ and Ca were calculated and compared with Freidman/Wilcoxon test. Serum concentrations were also categorized according to Canadian Society of Nephrology (CSN) guidelines target values for pre and clinic (post-post) samples. The proportion of patients that would be categorized differently by clinic (post-post) samples was determined. Results: There was a significant difference between pre-serum values compared to post and clinic (post-post) values. Overall, $\mathrm{iPTH}, \mathrm{PO}_{4}$ and Ca values would be misclassified in $25 \%, 70 \%$ and $50 \%$, respectively if blood was drawn at the clinic visit (post-post) compared to pre-HD as per CSN guidelines. Conclusions: Although no specific guideline has been written for NHHD patients, to ensure consistency of management compared to in-centre HD patients, lab values should be drawn pre-HD until clinical evidence suggests that the recommendations should be different for NHHD.
\end{abstract}

Keywords: Nocturnal Hemodialysis; Calcium; Phosphate; Parathyroid Hormone

\section{Introduction}

Conventional in-centre hemodialysis (HD) is the most common dialysis modality used to treat patients with end-stage renal disease (ESRD). Despite the widespread acceptance of conventional in-centre HD as the standard of care, patients experience a poor quality of life and have a higher mortality rate compared to general population [1]. In an attempt to reduce morbidity and mortality, a few novel approaches that increase dialysis frequency and/or time have been introduced. Nocturnal home HD is one such modality in which patients typically undergo HD for 3 - 6 nights per week for greater than 5 hours per treatment. This type of HD is associated with an improvement in blood pressure, regression of left ventricu-

\footnotetext{
*Conflicts of interest: None of the investigators have any conflicts of interest with respect to this study. The manuscript has not been published previously in whole or in part except in abstract form (WCN 2011).
}

lar hypertrophy [2] and an improvement in quality of life [3]. Improvements in the control of mineral metabolism parameters such as a decrease in serum phosphate and decrease in the use phosphate binders have also been reported [2,4].

Despite the benefits of nocturnal home hemodialysis, this modality is not without its own challenges for both patients and healthcare providers [5]. One such challenge is the timing of blood draw for laboratory analysis and clinician interpretation of the results. Some HD guidelines have emphasized that target levels for bone mineral markers such as calcium $(\mathrm{Ca})$, phosphate $\left(\mathrm{PO}_{4}\right)$ and intact parathyroid hormone (iPTH) be based on samples drawn pre-HD [6]. Since no specific guidelines regarding optimal timing of blood sampling has been published for home nocturnal HD patients, most programs encourage pre-post HD blood collection to be done at home. However, there is variability in this practice pattern in different Canadian centres providing care for nocturnal home 
HD patients based on a Canadian Practice patterns survey. Of the 17 program directors who responded to our survey, a centrifuge was not supplied by 3 programs and a further 3 programs only sometimes provide centrifuges (not published). The reasons cited included cost and patient inconvenience/preference.

To our knowledge, no study has been performed to determine if different clinical decisions would be made in the management of mineral metabolism abnormalities based on the timing of blood sample draw in relationship to the HD treatment in nocturnal home HD patients. Therefore, we conducted a prospective cohort study to examine the variability in pre, post and clinic (post-post) serum values for $\mathrm{Ca}, \mathrm{PO}_{4}$ and $\mathrm{iPTH}$ in nocturnal home hemodialysis patients to determine if they were statistically and/or clinically different. The study was approved by The Ottawa Hospital Research Ethics Board.

\section{Materials and Methods}

Twenty prevalent adult nocturnal home HD patients from our tertiary care centre participated in the study. Patients included were over 18 years old and treated for at least 18 hours/week HD (minimum 6 hours per treatment, 3 days per week). Patients with a successful parathyroiddectomy (iPTH less that $10 \mathrm{pmol} / \mathrm{L}$ ) were excluded.

After signing informed consent, patients collected blood pre and post their usual Tuesday nocturnal home HD session. Pre-dialysis samples are collected directly from the arterial needle or catheter lumen prior to initiation of treatment. For post-dialysis samples immediately prior to rinseback, the dialysate flow and the ultrafiltration are turned off. Blood pump speed is reduced to 100 $\mathrm{mls} / \mathrm{min}$ for 15 seconds; the blood sample is drawn with a vacutainer needle and tube directly from the arterial port. The samples were centrifuged and stored in the patient's fridge overnight. An additional blood sample was collected in clinic on Wednesday (clinic sample or postpost). All samples were analyzed in the Ottawa Hospital laboratory in accordance with the Ottawa Hospital laboratory guidelines. iPTH was analyzed with Beckman Coulter Immunoassay analyzer (coefficient of variation for iPTH; $6.9 \%$ at $2.6 \mathrm{pmol} / \mathrm{L}, 6.9 \%$ at $19.9 \mathrm{pmol} / \mathrm{L}$ and $5.8 \%$ at $59.3 \mathrm{pmol} / \mathrm{L})$. $\mathrm{Ca}$ and $\mathrm{PO}_{4}$ were analyzed with Siemens Vista 1500 analyzer (coefficient of variation for $\mathrm{Ca} ; 2.63 \%$ at $1.42 \mathrm{mmol} / \mathrm{L}$, and $2.1 \%$ at $2.5 \mathrm{mmol} / \mathrm{L}$, coefficient of variation for $\mathrm{PO}_{4} ; 3.4 \%$ at $0.6 \mathrm{mmol} / \mathrm{L}$ and $2.4 \%$ at $1.2 \mathrm{mmol} / \mathrm{L}$ ).

Median and interquartile range of pre, post and clinic (post-post) values of iPTH, $\mathrm{PO}_{4}$ and $\mathrm{Ca}$ were determined. The difference in pre, post and clinic (post-post) samples was examined with Freidman/Wilcoxon test. We also categorized values in each sample of pre and clinic (postpost), as within target and out of target as per Canadian Society of Nephrology guidelines and determined the proportion of patients that were categorized differently by clinic (post-post) samples.

\section{Results}

The majority of patients were male (60\%) with a mean age of 47 years (Table 1). The median hemodialysis treatment time was 7.5 hours (IQR 6 - 8 hrs) for the study session and the median interval between pre-HD bloodwork, and clinic (post-post) HD bloodwork was 11.7 hours (IQR 10.7 - 14 hrs). Median time from post-HD bloodwork and post-post HD blood sampling was 5.3 hours (IQR 2.9 - 6.2 hours). Calcium concentration in the dialysate ranged between 1.25 and $1.75 \mathrm{mmol} / \mathrm{L}$ (mean $1.45 \mathrm{mmol} / \mathrm{L})$. Six patients (30\%) had phosphate added to their dialysate and 6 patients (30\%) were taking phosphate binders; either Calcium carbonate or Sevelamer. Nine patients (45\%) were taking Calcitriol and 2 patients were also taking Cinacalcet. The majority of patients (9 out of 20,55\%) were using the Fresenius Optiflux 160 or 200 dialyzer, 6 patients (30\%) were using the Fresenius F16 dialyzer and 1 patient each (5\%) was using the Baxter Exeltra 190 and the Fresenius F6 dialyzer.

Median and interquartile range of pre, post and clinic (post-post) values of iPTH, $\mathrm{PO}_{4}$ and $\mathrm{Ca}$ are shown in Figures 1-3. Median pre-HD $\mathrm{Ca}, \mathrm{PO}_{4}$, iPTH values were significantly different compared to median postHD calcium, phosphate and iPTH values respectively. The same was true comparing median pre-HD values with clinic (post-post) values (Table 2).

The serum values of iPTH, $\mathrm{PO}_{4}$ and $\mathrm{Ca}$ were also clinically different depending on when the blood sample was drawn (Tables 3-8). Overall in our 20 NHHD patients, iPTH, PO4, Ca and values would be misclassified in $25 \%, 70 \%$ and $50 \%$ respectively if blood was drawn at the clinic visit (post-post) compared to pre-HD as per target values based on Canadian Society of Nephrology mineral metabolism guidelines. Similarly, if blood was only drawn post-HD, iPTH, $\mathrm{PO}_{4}$, and Ca values would be misclassified in $40 \%, 80 \%$ and $45 \%$ compared to preHD values.

Table 1. Baseline characteristics.

$\begin{array}{cc}\text { Age (years) } & 47.1 \text { (13.9) } \\ \text { Gender (M:F) } & 12: 8 \\ \text { Cause of ESRD (“N”) } & \text { GN (9), HTN (2), DM (1), } \\ \text { Other (4), Unknown (4) } & 3(4), 4(3) \\ \text { Number of treatments per week (“N”) } & 5(6), 6(7) \\ \text { Blood pump speed (mls/min) } & 338(51) \\ \text { Dialysate flow (mls/min) } & 350(83) \\ \text { Treatment duration in hours (“N”) } & 6(6), 7(1) \\ \end{array}$


Table 2. Median and interquartile range of pre, post and clinic (post-post) values of iPTH, $\mathrm{PO}_{4}$ and Ca.

\begin{tabular}{ccccc}
\hline & $\begin{array}{c}\text { Pre HD } \\
\text { median, IQR }\end{array}$ & $\begin{array}{c}\text { Post HD } \\
\text { median, IQR }\end{array}$ & $\begin{array}{c}\text { Post post HD } \\
\text { median, IQR }\end{array}$ & $\begin{array}{c}\text { Pre HD vs } \\
\text { post post HD }\end{array}$ \\
\hline $\mathrm{Ca}$ & $2.27(2.12-2.32)$ & $2.40(2.24-2.51)$ & $2.41(2.27-2.46)$ & $<0.001$ \\
$\mathrm{PO}_{4}$ & $1.62(1.18-2.06)$ & $0.82(0.73-1.12)$ & $1.00(0.76-1.27)$ & $<0.001$ \\
$\mathrm{iPTH}$ & $44.65(27.60-70.75)$ & $19.1(8.85-30.5)$ & $25.1(15.52-38.15)$ & $<0.001$ \\
\hline
\end{tabular}

Table 3. Pre and clinic (post-post) iPTH categorized as within target or out of target as per Canadian society of nephrology guidelines and the proportion of patients in each category.

\begin{tabular}{cccc}
\hline & Low post post HD iPTH & Normal post post HD iPTH & High post post HD iPTH \\
\hline Low preHD iPTH (<10.6 pmol/L) & $\mathbf{1 0 \%}$ & $0 \%$ & $0 \%$ \\
Normal preHD iPTH (10.6 - 53 pmol/L) & $0 \%$ & $\mathbf{5 5 \%}$ & $0 \%$ \\
High preHD iPTH (>53 pmol/L) & $0 \%$ & $25 \%$ & $\mathbf{1 0 \%}$ \\
\hline
\end{tabular}

Table 4. Pre and clinic (post-post) $\mathrm{PO}_{4}$ categroized as within target or out of target as per Canadian society of nephrology guidelines and the proportion of patients in each category.

\begin{tabular}{|c|c|c|c|}
\hline & Low post post $\mathrm{HD} \mathrm{PO}_{4}$ & Normal post post $\mathrm{HD} \mathrm{PO}_{4}$ & High post post $\mathrm{HD} \mathrm{PO}_{4}$ \\
\hline Low preHD $\mathrm{PO}_{4}(<0.81 \mathrm{mmol} / \mathrm{L})$ & $0 \%$ & $0 \%$ & $0 \%$ \\
\hline Normal preHD $\mathrm{PO}_{4}(0.81-1.58 \mathrm{mmol} / \mathrm{L})$ & $25 \%$ & $15 \%$ & $0 \%$ \\
\hline High preHD $\mathrm{PO}_{4}(>1.58 \mathrm{mmol} / \mathrm{L})$ & $0 \%$ & $45 \%$ & $15 \%$ \\
\hline
\end{tabular}

Table 5. Pre and clinic (post-post) Ca categorized as within target or out of target as per Canadian society of nephrology guidelines and the proportion of patients in each category.

\begin{tabular}{cccc}
\hline & Low post post HD Ca & Normal post post HD Ca & High post post HD Ca \\
\hline Low preHD Ca $(<2.2 \mathrm{mmol} / \mathrm{L})$ & $5 \%$ & $30 \%$ & $0 \%$ \\
Normal preHD Ca $(2.2-2.52 \mathrm{mmol} / \mathrm{L})$ & $0 \%$ & $45 \%$ & $20 \%$ \\
High preHD Ca $(>2.52 \mathrm{mmol} / \mathrm{L})$ & $0 \%$ & $0 \%$ & $0 \%$ \\
\hline
\end{tabular}

Table 6. Pre and post iPTH categorized as within target and out of target as per Canadian society of nephrology guidelines and the proportion of patients in each category.

\begin{tabular}{cccc}
\hline & Low post HD iPTH & Normal post HD iPTH & High post HD iPTH \\
\hline Low preHD iPTH $(<10.6 \mathrm{pmol} / \mathrm{L})$ & $10 \%$ & $0 \%$ & $0 \%$ \\
Normal preHD iPTH $(10.6-53 \mathrm{pmol} / \mathrm{L})$ & $15 \%$ & $35 \%$ & $5 \%$ \\
High preHD iPTH $(>53 \mathrm{pmol} / \mathrm{L})$ & $5 \%$ & $15 \%$ & $15 \%$ \\
\hline
\end{tabular}

Table 7. Pre and post $\mathrm{PO}_{4}$ categorized as within target and out of target as per Canadian society of nephrology guidelines and the proportion of patients in each category.

\begin{tabular}{|c|c|c|c|}
\hline & Low post $\mathrm{HD} \mathrm{PO}_{4}$ & Normal post $\mathrm{HD} \mathrm{PO}_{4}$ & High post $\mathrm{HD} \mathrm{PO}_{4}$ \\
\hline Low preHD $\mathrm{PO}_{4}(<0.81 \mathrm{mmol} / \mathrm{L})$ & $0 \%$ & $0 \%$ & $0 \%$ \\
\hline Normal preHD $\mathrm{PO}_{4}(0.81$ - $1.58 \mathrm{mmol} / \mathrm{L})$ & $25 \%$ & $15 \%$ & $0 \%$ \\
\hline High preHD $\mathrm{PO}_{4}(>1.58 \mathrm{mmol} / \mathrm{L})$ & $25 \%$ & $30 \%$ & $5 \%$ \\
\hline
\end{tabular}


Table 8. Pre and post Ca categoriezed as within target and out of target as per Canadian society of nephrology guidelines and the proportion of patients in each category.

\begin{tabular}{cccc}
\hline & Low post HD Ca & Normal post HD Ca & High post HD Ca \\
\hline Low preHD Ca $(<2.2 \mathrm{mmol} / \mathrm{L})$ & $\mathbf{1 0 \%}$ & $25 \%$ & $5 \%$ \\
Normal preHD Ca $(2.2-2.52 \mathrm{mmol} / \mathrm{L})$ & $0 \%$ & $\mathbf{4 5 \%}$ & $15 \%$ \\
High preHD Ca $(>2.52 \mathrm{mmol} / \mathrm{L})$ & $0 \%$ & $0 \%$ & $\mathbf{0 \%}$ \\
\hline
\end{tabular}

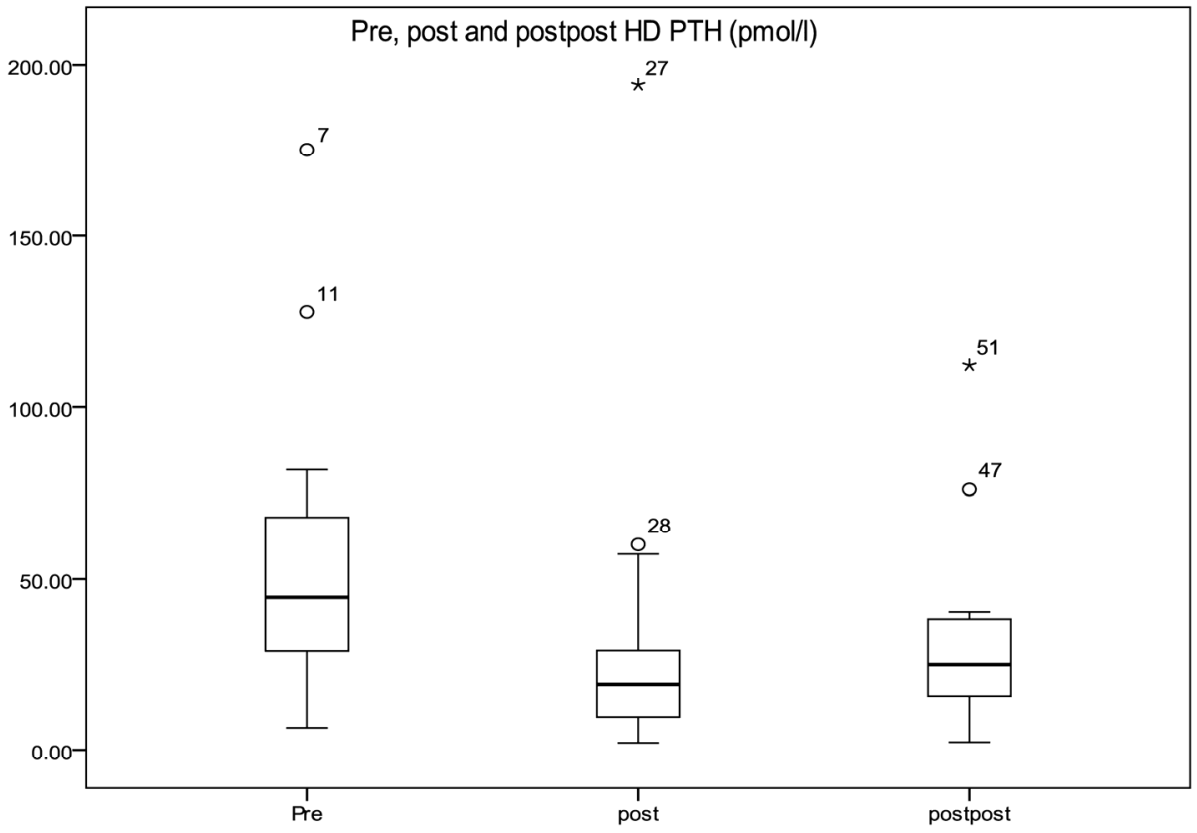

Figure 1. Median and interquartile range of pre, post and clinic (post-post) values of iPTH (outliers are shown as circles).

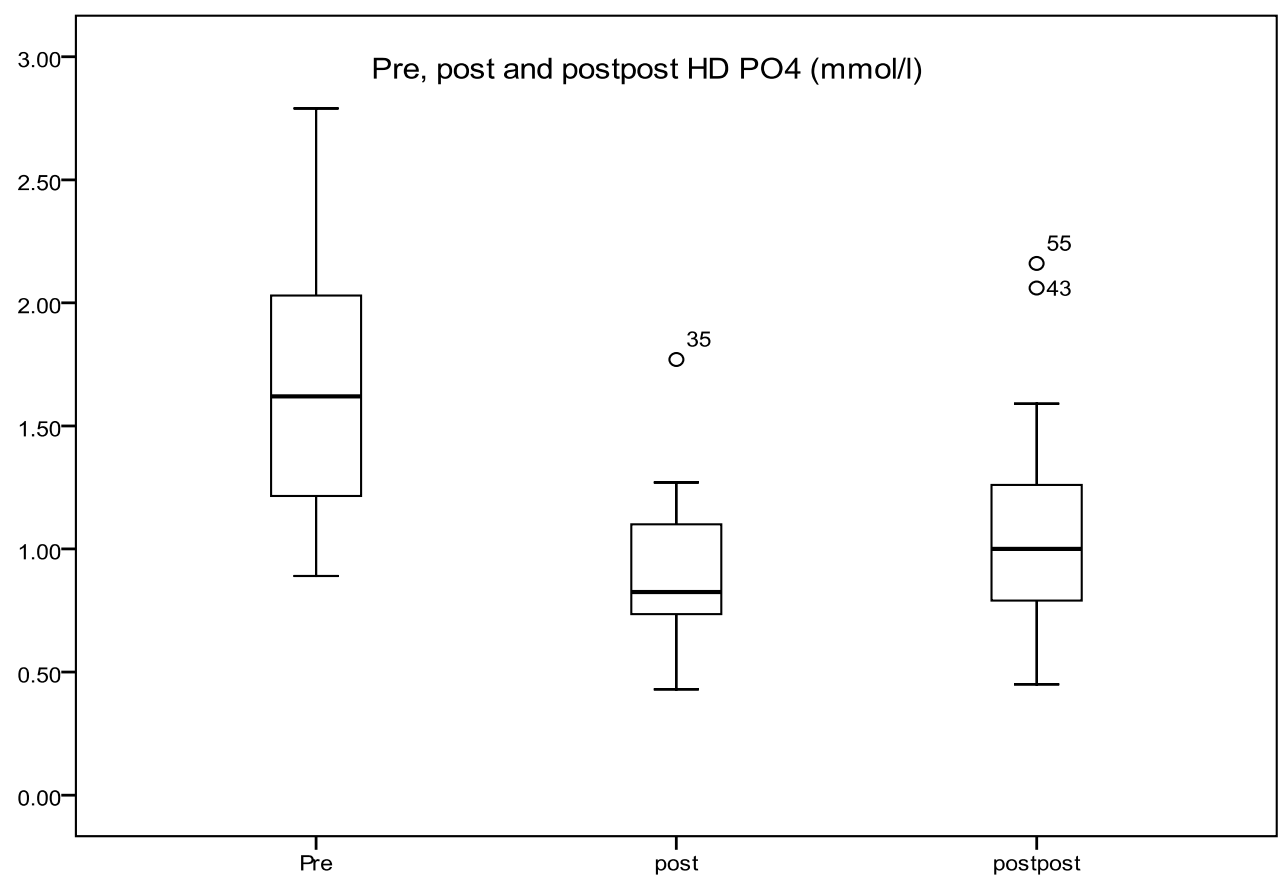

Figure 2. Median and interquartile range of pre, post and clinic (post-post) values of $\mathrm{PO}_{4}$ (outliers are shown as circles). 


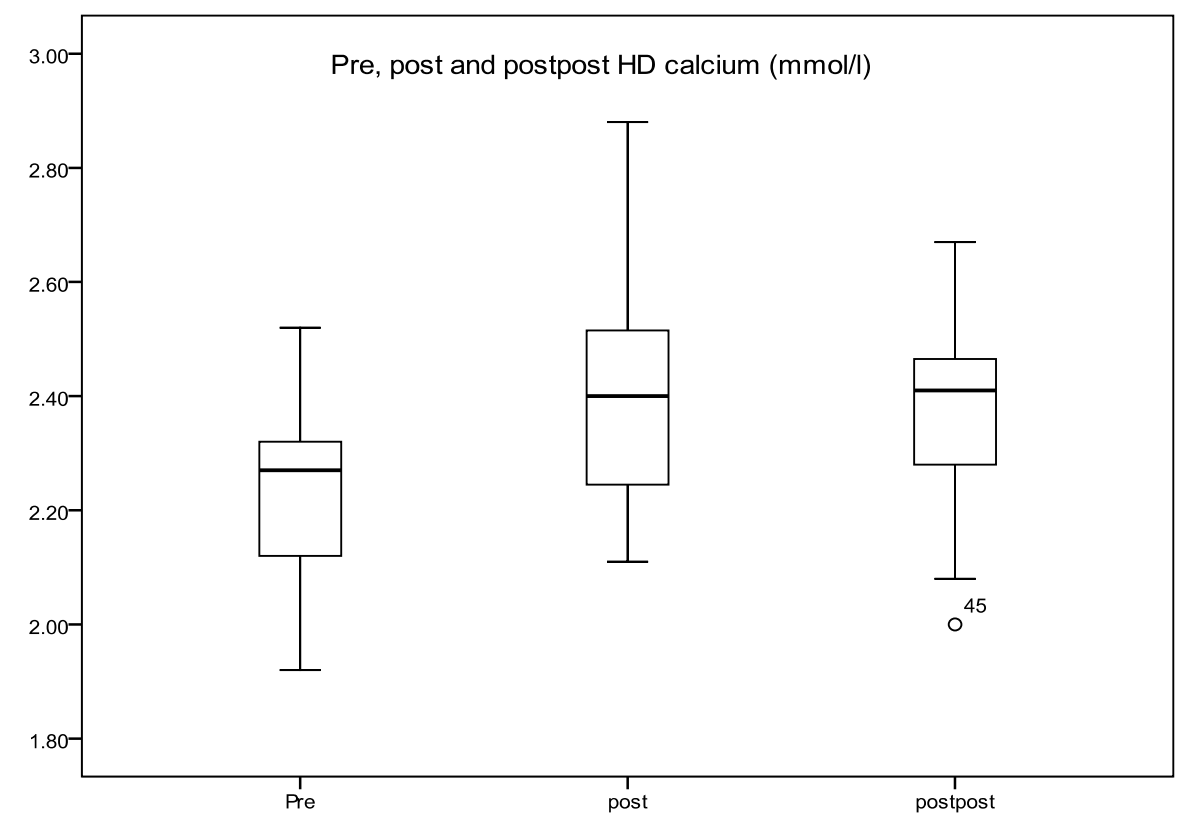

Figure 3. Median and interquartile range of pre, post and clinic (post-post) values of Ca (outliers are shown as circles).

\section{Discussion}

To our knowledge, this is the first study to compare values of commonly measured mineral metabolism markers pre, post and post-post (clinic visit) dialysis treatment in nocturnal home hemodialysis patients. We have demonstrated that the timing of blood collection has profound effects on the serum concentration of $\mathrm{Ca}, \mathrm{PO}_{4}$ and iPTH in nocturnal home hemodialysis patients that are both clinically and statistically significant. Interventions for the management of mineral metabolism may be applied differently depending on when patients collect their blood samples. This has relevance for patient management but may also affect the interpretation of the results of observational studies in which the timing of blood sample collection may be variable.

Abnormalities in serum calcium, phosphate and parathyroid hormone are common in dialysis dependent patients and constitute part of a systemic syndrome known as chronic kidney disease bone and mineral disorder [7]. An increased risk of fracture, vascular calcification and hospitalization has been associated with altered values of bone metabolism markers [8-10]. Patients with ESRD treated with conventional HD who have abnormal markers of mineral metabolism also appear to be at increased risk for all cause mortality and cardiovascular mortality [10,11].

In an attempt to decrease morbidity and mortality related to mineral metabolism disturbances, clinical practice guidelines (CPG) have been published by the National Kidney Foundation [12], Canadian Society of Nephrology [13], the United Kingdom [14], Caring for Australians with Renal Impairment (CARI) [15], Kidney
Disease: Improving Global Outcomes (KDIGO) [16], Renal Association Clinical Practice Guideline [6] and also by the European Best Practice Society [17]. Acceptable values for biochemical markers have been specified; however no discrimination has been made between patients on different dialysis prescriptions. Only some of the guidelines have included a specified timing for blood collection. For example in the CARI guideline, it is recommended that for serum calcium and phosphate measurement, a predialysis blood sample should be sent [15]. The Renal Association Clinical Practice Guideline has also recommended pre-dialysis blood sampling for biochemical and haematological measurements [6]. Similarly not all studies linking different target values for bone metabolism markers to clinical outcomes have clearly specified the timing of blood sampling in their studied population [18,19].

Despite these limitations, to our knowledge blood collection for $\mathrm{Ca}, \mathrm{PO}_{4}$ and $\mathrm{iPTH}$ for in-centre hemodialysis patients is typically done predialysis, midweek. However, this may not be true in home nocturnal hemodialysis patients based on a Canadian survey of practice patterns (not published). Although bone mineral metabolism markers have been studied in nocturnal hemodialysis patients, limited data exists in which different target values for $\mathrm{Ca}, \mathrm{PO}_{4}$ and iPTH have been examined with respect to clinically important outcomes such as mortality, vascular calcification or fracture in this patient population. However manipulation of the dialysate in nocturnal HD, compared to conventional HD, is common which may have an affect on serum values depending on the timing of the blood sample collection. The dialysate cal- 
cium is often increased above $1.25 \mathrm{mmol} / \mathrm{L}$ in order to treat worsening hyperparathyroidism and falling bone mineral density such that hypercalcemia may be present immediately post dialysis [20-21]. This is consistent with our finding that $20 \%$ of patients had a high serum calcium post dialysis whether the blood was drawn immediately post dialysis or in the clinic. The transiently high calcium would be expected to suppress serum PTH values [20]. This is consistent with the $35 \%$ of patients with a PTH value in a lower category on the post compared to the pre-HD blood work. The longer duration of dialysis associated with nocturnal has been shown to significantly reduce serum phosphate levels and in many reported studies, phosphate has been added to the dialysate. Serum phosphate values would be misclassified as much at $80 \%$ of the time if only a post-HD value is used based on our study results.

A CPG has not been specifically written for patients on longer dialysis duration to define optimal mineral metabolism values or suggestions for timing of blood collection that address the uncertainties that exist in target values and the special challenges for patients who undertake their dialysis at home. Our study would suggest that the timing of blood collection has relevance for the interpretation and management of mineral metabolism for nocturnal home hemodialysis patients. The interpretation of studies that are based on this patient population may also be challenging if the timing of blood collection has not been standardized. Although one could argue that the "right numbers" and the "right time" are not known, ultimately the clinician must make treatment decisions on mineral metabolism management that are likely to be altered by timing of blood sample collection such that consistency across renal programs would be desirable.

Our study has a number of limitations. The small patient numbers with different dialysis prescriptions including frequency and duration of the HD treatment, different intervals between pre, post and post-post samples, type of dialyzer and dialysate additives, different medication including phosphate binders and PTH suppressors, all have an impact on the mineral metabolism markers that were examined. However this represents a real patient population with all of the variables that can be manipulated in an attempt to manage mineral metabolism abnormalities in ESRD patients treated with HD. Our study also does not address the potential usefulness of measuring post dialysis serum calcium and phosphate in guiding decisions about dialysis additives. Despite these limitations we believe our sample represents nocturnal home hemodialysis patients of other centres and we have identified the high likelihood of misclassifying patients and making different treatment decisions depending on the timing of the blood sample draw.
In conclusion until outcome data on mineral metabolism management are available in nocturnal hemodialysis patients, for consistency, blood samples should be drawn pre-dialysis similar to in-centre HD patients. This recommendation does not take into account the added convenience to patients of having blood samples drawn in clinic or the potential reduction in costs associated with not providing patients with centrifuges in the home.

\section{Acknowledgements}

We would like to thank Jessica Wagner (research coordinator), the nurses in the home dialysis unit and the patients for their assistance with this study. Dr. D. Zimmerman receives salary support from the department of medicine at the Ottawa Hospital.

\section{REFERENCES}

[1] A. Laupacis, P. Keown, N. Pus, et al., "A Study of the Quality of Life and Cost-Utility of Renal Transplant,” Kidney International, Vol. 50, No. 1, 1996, pp. 235-242. doi:10.1038/ki.1996.307

[2] B. F. Culleton, M. Walsh, S. W. Klarenbach, et al., "Effect of Frequent Nocturnal Hemodialysis vs Conventional Hemodialysis on Left Ventricular Mass and Quality of Life: A Randomized Controlled Trial," Journal of the American Medical Association, Vol. 298, No. 11, 2007, pp. 1291-1299. doi:10.1001/jama.298.11.1291

[3] P. A. McFarlane, A. M. Bayoumi, A. Pierratos and D. A. Redelmeier, "The Quality of Life and Cost Utility of Home Nocturnal and Conventional in-Center Hemodialysis,” Kidney International, Vol. 64, No. 3, 2003, pp. 10041011. doi:10.1046/j.1523-1755.2003.00157.x

[4] M. V. Rocco, R. S. Lockridge Jr, G. J. Beck, et al., “The Effects of Frequent Nocturnal Home Hemodialysis: The Frequent Hemodialysis Network Nocturnal Trial,” Kidney International, Vol. 80, No. 10, 2011, pp. 1080-1091. doi:10.1038/ki.2011.213

[5] M. John and K. Michael, "Starting a Home Hemodialysis Program,” Seminars in Dialysis, Vol. 20, No. 1, 2007, pp. 35-39. doi:10.1111/j.1525-139X.2007.00239.X

[6] R. Mactier, N. Hoenich, C. Breen, "Renal Assocition Clinical Practice Guidelines 5th Edition 2009-2012,” Nephron Clinical Practice, Vol. 118, Suppl. 1, 2011, pp. 241-286.

[7] S. Moe, T. Drueke, J. Cunningham, et al., "Definition, Evaluation, and Classification of Renal Osteodystrophy: A Position Statement from Kidney Disease: Improving Global Outcomes (KDIGO),” Kidney International, Vol. 69, No. 11, 2006, pp. 1945-1953. doi:10.1038/sj.ki.5000414

[8] W. G. Goodman, J. Goldin, B. D. Kuizon, et al., "Coronary Artery Calcification in Young Adults with EndStage Renal Disease Who Are Undergoing Dialysis," The New England Journal of Medicine, Vol. 342, No. 20, 2000, pp. 1478-1483. doi:10.1056/NEJM200005183422003

[9] W. Y. Qunibi, C. A. Nolan and J. C. Ayus, "Cardiovas- 
cular Calcification in Patients with End-Stage Renal Disease: A Century Old Phenomenon,” Kidney International, Vol. 62, Suppl. 82, 2002, pp. S73-S80. doi:10.1046/j.1523-1755.62.s82.15.x

[10] G. A. Block, P. S. Klassen, J. M. Lazarus, N. Ofsthun, E. G. Lowrie and G. M. Chertow, "Mineral Metabolism, Mortality, and Morbidity in Maintenance Hemodialysis," Journal of the American Society of Nephrology, Vol. 15, No. 8, 2004, pp. 2208-2218. doi:10.1097/01.ASN.0000133041.27682.A2,

[11] M. L. Melamed, J. A. Eustace, L. Plantinga, et al., "Changes in Serum Calcium, Phosphate, and PTH and the Risk of Death in Incident Dialysis Patients: Along Longitudinal Study,” Kidney International, Vol. 70, 2006, pp. 351-357. doi:10.1038/sj.ki.5001542

[12] National Kidney Foundation, "K/DOQI Clinical Practice Guidelines for Bone Metabolism and Disease in Chronic Kidney Disease,” American Journal of Kidney Diseases, Vol. 42, Suppl. 3, 2003, pp. S1-S201. doi:10.1016/S0272-6386(03)00553-5

[13] K. Jindal, C. T. Chan, C. Deziel, et al., "Hemodialysis Clinical Practice Guidelines for the Canadian Society of Nephrology," Journal of the American Society of $\mathrm{Ne}$ phrology, Vol. 17, No. 3, 2006, pp. S1-S27.

[14] Joint Specialty Committee on Renal Medicine of the Royal College of Physicians and the Renal Association, and the Royal College of General Practitioners, "Chronic Kidney Disease in Adults: UK Guidelines for Identification, Management and Referral,” Royal College of Physicians, London, 2006.

[15] G. Elder, R. Faull, P. Branley and C. Hawley, "Management of Bone Disease, Calcium, Phosphate and Parathyroid Hormone," Nephrology, Vol. 11, Suppl. 1, 2006, pp.
S230-S261. doi:10.1111/j.1440-1797.2006.00508.x

[16] CKD-MBD Work Group, "KDIGO Clinical Practice Guideline for the Diagnosis, Evaluation, Prevention, and Treatment of Chronic Kidney Disease-Mineral and Bone Disorder (CKD-MBD),” Kidney International, Vol. 113, 2009, pp. S1-130.

[17] J. B Cannata-Andía, T. B. Drueke, J. Cunningham, et al., "Clinical Algorithms on Renal Osteodystrophy," Nephrology Dialysis Transplantation, Vol. 15, Suppl. 5, 2000, pp. S39-S57.

[18] F. Tentori, M. J. Blayney, J. M. Albert, et al., "Mortality Risk for Dialysis Patients with Different Levels of Serum Calcium, Phosphorus, and PTH: The Dialysis Outcomes and Practice Patterns Study (DOPPS),” American Journal of Kidney Diseases, Vol. 52, No. 3, 2008, pp. 519-553. doi:10.1053/j.ajkd.2008.03.020

[19] G. A. Block, P. S. Klassen, J. M. Lazarus, N. Ofsthun, E. G. Lowrie and G. M. Chertow, "Mineral Metabolism, Mortality, and Morbidity in Maintenance Hemodialysis," Journal of the American Society of Nephrology, Vol. 15, No. 8, 2004, 2208-2218. doi:10.1097/01.ASN.0000133041.27682.A2

[20] R. M. Lindsay, F. Alhejaili, G. Nesrallah, et al., "Calcium and Phosphate Balance with Quotidian Hemodialysis," American Journal of Kidney Diseases, Vol. 42, No. 1, 2003, pp. S24-S29. doi:10.1016/S0272-6386(03)00534-1

[21] N. Toussaint, J. Boddington, R. Simmonds, C. Waldron, C. Soerville and J. Agar, "Calcium Phosphate Metabolism and Bone Mineral Density with Nocturnal Hemodialysis,” Hemodialysis International, Vol. 10, No. 3, 2006, pp. 280-286. doi:10.1111/j.1542-4758.2006.00109.x 